\title{
Coalitional Public Announcement Games
}

\author{
Thomas Ågotnes ${ }^{1}$ and Hans van Ditmarsch ${ }^{2}$ \\ 1 University of Bergen, Norway, thomas . agotnes e infomedia.uib. no \\ ${ }^{2}$ University of Sevilla, Spain, \& IMSc, Chennai, India, hvd@us .es
}

\begin{abstract}
Dynamic epistemic logic (DEL) is a popular framework for studying interaction in multi-agent systems. DELs describe the actions available to the agents, and their epistemic pre- and post-conditions. By extending standard epistemic models with preferences over epistemic states, we can say something about rational behaviour as well, combining logic and game theory. In this paper we assume that preferences are represented by epistemic goal formulae, and actions are public announcements as described by public announcement logic. We are interested in analysing coalition formation and in particular coalitional stability in such settings. To this end, we describe how such epistemic goal models can be viewed as coalitional (cooperative) games, and study and characterise the resulting class of games and their solutions in different ways. We use a model of coalitional games under imperfect information that is more natural for many logical and computational settings than most existing models, and propose some related solution concepts extending the notion of the core under common knowledge.
\end{abstract}

\section{Introduction}

Epistemic logic is a widely popular approach for reasoning about knowledge and information in multi-agent systems $[10,15]$. One of the most prominent recent developments is dynamic epistemic logic (DEL) [22]. Information is communicated, so knowledge is by no means static, and DEL is an umbrella term for extensions of epistemic logic with dynamic operators to formalise reasoning about information change. The perhaps simplest and best understood DEL is public announcement logic (PAL) [19,11]. Dynamic epistemic logic is used to describe and analyse epistemic pre- and post- conditions of actions; which actions are available to an agent and how they will affect the knowledge states of the agents in the system. However, until recently $[4,2]$ there has been very little work on analysing "what will happen" - how will (or should) rational agents in such scenarios actually interact? Rational interaction has, of course, been widely studied in game theory, another, in addition to logic, key theoretical foundation of multi-agent systems. And almost everything is already in place in DEL for a game theoretic analysis: individual actions, outcomes, and the relationship between the two. This model can easily be extended with the missing piece of the puzzle: agents' preferences over outcomes, i.e., over epistemic states. [4] argues that by varying the types of actions/events considered, representation of preferences, and assumptions about game-theoretic interaction (coalitional, non-cooperative, extensive form, etc.), we get a rich framework for studying rational information change. In [4] one particular case is studied in some detail: actions are truthful public announcements, preferences are represented by epistemic 
goal formulae in the style of Boolean games [18, 12], and agents are self-interested and act simultaneously like in non-cooperative strategic form games. [2] studies a similar setting, where the difference is that actions are questions that are obliged to be truthfully answered. In the current paper we change the game-theoretic assumption: we assume that it is typically the case that an agent cannot satisfy her goals by herself, but must cooperate with others, and we are interested in well-known related concepts such as coalitional stability and the core. Thus, in this paper we study the coalitional (or cooperative), rather than non-cooperative, games, inherent in epistemic models. Like in [4] we assume that preferences are represented by epistemic goal formulae, and that actions are public announcements. Issues in the intersection of logic and coalitional game theory have been studied before $[9,3]$, but we are not aware of any work on the coalitional game theoretic properties of PAL or other DELS.

In the coalitional games we study in this paper the coalitions' possible actions, joint public announcements, are defined by the agents' knowledge as described by models of epistemic logic. But since one agent does not necessarily know what another agent knows and thus what he can announce, we need a model of coalitional games under imperfect information. While coalitional games with imperfect information have received far less attention in the literature than non-cooperative games with imperfect information, with Bayesian games [13] being the main model, they have received some attention lately $[7,16,14,8]$. However, these existing models of imperfect information coalitional games don't capture our logical setting well. Similarly to Bayesian games, most models assume that agents beliefs are represented as probability distribution, but that information is not available in the epistemic structures we want to induce the game models from. An interesting recent work which does not make that assumption is [8]. However, [8] instead assumes that an agent's beliefs are represented by a "best guess" about other agents types (beliefs, etc.) rather than a set of alternatives, and this model is not rich enough for our purposes. In this paper we therefore define a "new" model of coalitional games under imperfect information, which is extremely straightforward and general, and natural from a computer science perspective: an epistemic state space with a coalitional game associated with each state.

We define these epistemic coalitional game structures, and discuss some related solution concepts refining the notion of the core using common knowledge, in Section 4. In Section 5 we induce coalitional games from epistemic goal structures, and in Section 6 we study the core of the induced games, including the relationship between the core and properties of the goal formulae. This is an analysis of games about logic, games where, e.g., both actions and preferences are defined in terms of logical formulae. There is currently a considerable interest in research in the other direction, logic about games, in particular modal logics interpreted in the context of games which are able to express properties such as solution concepts [21] - also for coalitional games [3]. In this paper we are interested in this approach as well, for our games (i.e., in logic about games about logic!). It turns out (Section 7) that Group Announcement Logic [1], an extension of PAL, can capture several solution concepts for coalitional games. Not all coalitional games correspond to epistemic goal structures. In Section 8, we give a complete (up to bisimulation) characterisation of the class of finite games that do. In addition we prove a compactness result that shows that epistemic structures are a compact representation 
of such games. We discuss related and future work and conclude in Section 9. First, we briefly review some key concepts in logic and game theory, and introduce a new (as far as we know) notion of bisimulation for coalitional games in Section 3.

\section{Background}

Logic: Due to lack of space we must be very brief here. The language $\mathcal{L}_{\text {pal }}$ of public announcement logic (PAL) [19] over a set of agents $N=\{1, \ldots, n\}$ and an infinite set of primitive propositions $\Theta$ is defined as follows, where $i$ is an agent and $p \in \Theta$ :

$$
\varphi::=p\left|K_{i} \varphi\right| \neg \varphi\left|\varphi_{1} \wedge \varphi_{2}\right|\left[\varphi_{1}\right] \varphi_{2}
$$

We write $\left\langle\varphi_{1}\right\rangle \varphi_{2}$ ( $\varphi_{1}$ is true and $\varphi_{2}$ is true after $\varphi_{1}$ is announced) resp. $\hat{K}_{i} \varphi$ ( $i$ considers $\varphi$ possible) for the duals $\neg\left[\varphi_{1}\right] \neg \varphi_{2}$ and $\neg K_{i} \neg \varphi$. The trivial announcement is a an (arbitrary) tautology $T$. The purely epistemic fragment of the language, i.e., formulae not containing public announcement operators $[\varphi]$, is denoted $\mathcal{L}_{e l}$.

A epistemic (Kripke) structure over $N$ and $\Theta$ is a tuple $M=\left(S, \sim_{1}, \ldots, \sim_{n}, \pi\right)$ where $S$ is a set of states, $\sim_{i} \subseteq S \times S$ an epistemic indistinguishability relation assumed to be an equivalence relation for each agent $i$, and $\pi: \Theta \rightarrow 2^{S}$ assigns primitive propositions to the states in which they are true. A pointed epistemic structure is a pair $(M, s)$ where $s$ is a state in $M$. The interpretation of formulae in pointed epistemic structures is defined as follows (the other clauses are defined in the usual truth-functional way).

$$
\begin{aligned}
& M, s \models K_{i} \varphi \text { iff for every } t \text { such that } s \sim_{i} t, M, t \models \varphi \\
& M, s \models[\varphi] \psi \text { iff } M, s \models \varphi \text { implies that } M \mid \varphi, s \models \psi
\end{aligned}
$$

where $M \mid \varphi=\left(S^{\prime}, \sim_{1}^{\prime}, \ldots, \sim_{n}^{\prime}, \pi^{\prime}\right)$ such that $S^{\prime}=\left\{s^{\prime} \in S: M, s^{\prime}=\varphi\right\} ; \sim_{i}^{\prime}=\sim_{i}$ $\cap\left(S^{\prime} \times S^{\prime}\right) ; \pi^{\prime}(p)=\pi(p) \cap S^{\prime}$. Observe that $M, s=\langle\varphi\rangle \psi$ iff $M, s=\varphi$ and $M \mid \varphi, s=\psi$.

A recent development in the area of dynamic epistemic logic is to allow quantification over announcements (and other epistemic actions) [20, 5, 1]. Group Announcement Logic (GAL) [1] adds modal operators $\langle G\rangle$ to the PAL language, for any set $G$ of agents. $\langle G\rangle \varphi$ means that there is a truthful and public announcement that the members of $G$ can collectively make after which $\varphi$ will be true:

$$
M, s=\langle G\rangle \varphi \text { iff } \exists\left\{\psi_{i}: i \in G\right\} \subseteq \mathcal{L}_{e l} \text { s.t. } M, s \models\left\langle\bigwedge_{i \in G} K_{i} \psi_{i}\right\rangle \varphi .
$$

An important concept in the following is the notion of common knowledge. Something is common knowledge in a non-empty group $G$ in a state $s$ of an epistemic structure $M$, if it is true in all $G$-reachable states, i.e., in all states that are reachable from $s$ in $M$ by the transitive closure of the union of the indistinguishability relations for $G$. We shall sometimes extend the above logical languages with common knowledge operators $C_{G}$, where $M, s \models C_{G} \varphi$ holds iff $G$ has common knowledge of $\varphi$ in $s$.

Example 1. Consider two agents Ann and Bill. They both have a secret! Ann (1) knows that Helen is in love with Bill ( $p$ ) but is afraid to tell him that, i.e., Helen probably has not told Bill - but who knows. Whereas Bill (2) knows that Ann got through her maths exam $(q)$, although he reckons that she does not know that yet - he is unsure. 
In fact, $p$ and $q$ are both true. If we assume that Ann and Bill are commonly aware of these propositions, this uncertainty about each other can be visualized as follows in an (initial) pointed model $(M, t)$.

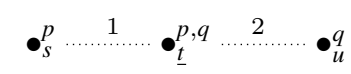

Ann and Bill can both make two different announcements. in state $t, K_{1} p$ is true, $K_{1} q$ is false, and $\left\langle K_{2} q\right\rangle K_{1} q$ is true. Also true in $t$ is $\langle 12\rangle\left(K_{1} q \wedge K_{2} p\right)$ : if Ann announces $p$ and Bill announces $q$, in the resulting model restriction we have $M\left|\left(K_{1} p \wedge K_{2} q\right), t\right|=$ $K_{1} q \wedge K_{2} p$. On the other hand, $\langle 1\rangle\left(K_{1} q \wedge K_{2} p\right)$ is false in $t$ : Anne can only make the trivial announcement, or announce her knowledge of $p$, and in neither model restriction she has learnt $q$. The following illustrates the different model restrictions and their dependencies. Note that in each model the trivial announcement is yet another option; which can be said to induce a reflexive arrow.

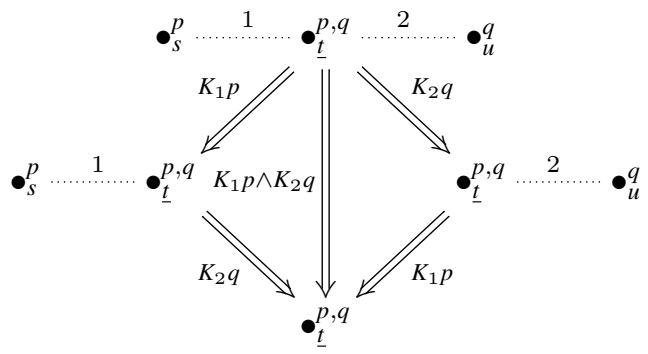

Coalitional Games: A coalitional game (without transferable utility) (see, e.g., [17, p. 268]) is a tuple $\Gamma=\left(N, \Omega, V, コ_{1}, \ldots, コ_{n}\right)$ where $N=\{1, \ldots, n\}$ is a non-empty set of agents; $\Omega$ a non-empty set of outcomes; $V:\left(2^{N} \backslash \emptyset\right) \rightarrow 2^{\Omega}$ the characteristic function, which defines the choices $V(C)$ available to every non-empty coalition $C$; and $\exists_{i} \subseteq \Omega \times \Omega$ a complete, reflexive, and transitive preference relation, for each $i \in N$.

We let $\omega \sqsupset_{i} \omega^{\prime}$ denote the fact that $\omega$ is strictly preferred over $\omega^{\prime}$ by agent $i$ (i.e., $\omega \sqsupseteq_{i} \omega^{\prime}$ but not $\left.\omega^{\prime} \sqsupseteq_{i} \omega\right)$. We sometimes refer to the set $N$ as the grand coalition. We will use $\mathcal{C}$ to denote the set of all non-empty coalitions $\left(2^{N} \backslash \emptyset\right)$. When $C=\{i\}$ is a singleton, we will sometimes abuse notation and write, e.g., $V(i)$ for $V(\{i\})$.

The most well known solution concept is the the core.

Definition 1 (The Core). The core of a coalitional game $\Gamma=\left(N, \Omega, V, \sqsupseteq_{1}, \ldots, \sqsupseteq_{n}\right)$ is the set of all $\omega \in V(N)$ such that there does not exist a coalition $C \subseteq N$ and $\omega^{\prime} \in V(C)$ for which $\omega^{\prime} \sqsupset_{i} \omega$ for all $i \in C$.

Intuitively, the outcomes in the core are "stable" in the sense that no coalition can be better off on their own. A key property of a coalitional game is whether or not the core is empty, if it is not, the grand coalition can be said to be "stable" in the sense that there is some outcome everyone can agree upon.

\section{Bisimulation for Coalitional Games}

When are two games essentially the same? A natural and common answer is when they can simulate each other; when for each move or choice made in one game there is a 
corresponding choice in the other, and the other way around. In this section, we define a notion of bisimulation for coalitional games. As far as we know, this has not appeared in the literature before.

For a reader familiar with the notion of bisimulation from logic, the key to understanding bisimulation on coalitional games is the following. In standard bisimulation on Kripke structures, bisimilar states must have the same values for the valuation function, which is essentially a unary "relation", while for the (typically) binary relation there only needs to be at least one corresponding choice in bisimilar states. For coalitional games, while $V$ is a unary "relation" on $\Omega$ and $\sqsupseteq_{i}$ is a binary relation, the roles are reversed: $V$ models the choices, while $\sqsupseteq_{i}$ models the properties of the outcomes (their utilities). This observation leads us to propose the following definition.

Definition 2 (Bisimulation). Let $\quad \Gamma^{1}=\left(N, \Omega^{1}, V^{1}, \sqsupseteq_{1}^{1}, \ldots, \sqsupseteq_{n}^{1}\right)$ and $\Gamma^{2}=\left(N, \Omega^{2}, V^{2}, \sqsupseteq_{1}^{2}, \ldots, \sqsupseteq_{n}^{2}\right)$ be coalitional games. A non-empty binary relation $Z \subseteq \Omega^{1} \times \Omega^{2}$ is a bisimulation between $\Gamma^{1}$ and $\Gamma^{2}$ iff the following hold.

1. (atoms) If $\omega_{1}^{1} Z \omega_{2}^{1}$ and $\omega_{1}^{2} Z \omega_{2}^{2}$, then

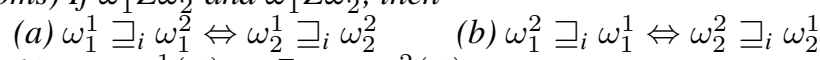

2. (forth) $\omega_{1} \in V^{1}(C) \Rightarrow \exists \omega_{2} \in V^{2}(C)$ s.t. $\omega_{1} Z \omega_{2}$

3. (back) $\omega_{2} \in V^{2}(C) \Rightarrow \exists \omega_{1} \in V^{1}(C)$ s.t. $\omega_{1} Z \omega_{2}$

$Z$ is a full bisimulation iff for every $\omega_{1} \in \Omega^{1}$ there is an $\omega_{2} \in \Omega^{2}$ such that $\omega_{1} Z \omega_{2}$, and for every $\omega_{2} \in \Omega^{2}$ there is an $\omega_{1} \in \Omega^{1}$ such that $\omega_{1} Z \omega_{2}$. If there is a full bisimulation between $\Gamma^{1}$ and $\Gamma^{2}$, we say that they are bisimulation equivalent.

The (atoms) condition says that agents should rank bisimilar outcomes in the same way (or, equivalently, assign the same utility to them). (forth) and (back) say that for every choice in one game, there is a corresponding choice in the other.

Note that (atoms) ensures that also strict preference is preserved:

Lemma 1. If $Z$ is a bisimulation between $\Gamma_{1}$ and $\Gamma_{2}$ s.t. $\omega_{1}^{1} Z \omega_{2}^{1}$ and $\omega_{1}^{2} Z \omega_{2}^{2}$, then $\omega_{1}^{1} \sqsupset_{i}$ $\omega_{1}^{2} \Leftrightarrow \omega_{2}^{1} \sqsupset_{i} \omega_{2}^{2}$ and $\omega_{1}^{2} \sqsupset_{i} \omega_{1}^{1} \Leftrightarrow \omega_{2}^{2} \sqsupset_{i} \omega_{2}^{1}$.

Key properties of coalitional games are invariant under bisimulation equivalence ${ }^{3}$ :

Theorem 1. Let $Z$ be a full bisimulation between $\Gamma_{1}$ and $\Gamma_{2}$ such that $\omega_{1} Z \omega_{2}$. Then $\omega_{1}$ is in the core of $\Gamma_{1}$ iff $\omega_{2}$ is in the core of $\Gamma_{2}$.

\section{Epistemic Coalitional Games}

As discussed in the introduction, we define a variant of coalitional games under imperfect information, that is natural and useful in a logical and computational setting.

Definition 3. An epistemic coalitional game structure (ECGS) is a tuple:

$$
\text { eg }=\left(S, N,\left\{\Gamma^{s}: s \in S\right\}, \sim_{1}, \ldots, \sim_{n}\right) \quad \text { where } \quad \Gamma^{s}=\left(N, \Omega^{s}, V^{s}, \sqsupseteq_{1}^{s}, \ldots, \beth_{n}^{s}\right)
$$

and $S$ is a set of states, $N$ a set of agents, $\Gamma^{s}$ a coalitional game for each $s$ in $S$, and for each $i, \sim_{i}$ is an epistemic indistinguishability relation, an equivalence relation, over $S$.

\footnotetext{
${ }^{3}$ Not only the core but also the bargaining set and the stable sets are invariant under this notion of bisimulation in a similar sense.
} 
This definition is very general, allowing us, e.g., to model agents who are ignorant about what the set of possible outcomes are and even what their own preferences are (although we will not make use of the full generality in the following).

What about solution concepts for ECGSs? Note that it might be the case that an outcome is in the core of a particular state in an ECGS, without one or several agents knowing it - it might be that an agent in that state considers another state possible where that outcome is not in the core. It is natural to use common knowledge to define epistemic coalitional solution concepts. There are several variants of common knowledge of interest here, for example common knowledge that the core is non-empty vs. common knowledge of why the core is non-empty (common knowledge that a particular element is in the core). Note that in connected structures, structures where any state is $\mathrm{N}$-reachable from any other state, common knowledge of all agents correspond to truth in all states in the model. For connected structures common knowledge of all agents is thus a model property rather than a pointed model property (said another way, a state has the property iff any other state has it). For simplicity we define the following properties for connected models only ${ }^{4}$. We identify the following ECGS properties:

1. Common knowledge of non-empty core: for every $s$, the core of $\Gamma^{s}$ is non-empty.

2. there is an $\omega$ which is in the core of $\Gamma^{s}$ for every $s \in S$

3. Common knowledge of the core: the core of $\Gamma^{s}$ is identical in every state $s$

We define the epistemic core of a connected ECGS to be the set of all $\omega \in \bigcap_{s \in S} \Omega^{s}$ such that $\omega$ is in the core of $\Gamma^{s}$ for every $s \in S$ (i.e., the intersection of the cores). Common knowledge of the core says that it is common knowledge what the outcomes in the core are. This is clearly a reasonable stability concept if the core is non-empty. However, it can be argued that for the grand coalition to be stable it is not necessary that the exact content of the core is common knowledge. It might be that there is at least one element that is commonly known to be in the core; non-empty epistemic core. But it might also be that in fact no element is commonly known to be in the core, but it is still commonly known that the core is non-empty; different agents might consider different cores possible but not that the core is empty. In this weakest case, common knowledge of non-empty core, the grand coalition is still stable in the sense that it common knowledge that there is some outcome everyone can agree upon - although it is not necessarily common knowledge what it is.

\section{From Epistemic Structures to Coalitional Games}

An epistemic goal structure (EGS) for $n$ agents is a tuple $G S=\left(M, \varphi_{1}, \ldots, \varphi_{n}\right)$ where $M$ is an epistemic structure over $N=\{1, \ldots, n\}$ and $\varphi_{1}, \ldots, \varphi_{n} \in \mathcal{L}_{e l}$ are goal formulae. A pointed EGS is a tuple $g s=\left(M, s, \varphi_{1}, \ldots, \varphi_{n}\right)$ where $\left(M, \varphi_{1}, \ldots, \varphi_{n}\right)$ is an EGS and $s$ is a state in $M$.

While PAL models arbitrary public announcements, we are here interested in "subjective" announcements, announcements made by some agent in the system. Such announcements will be of the form $K_{i} \varphi$. We assume that announcements are truthful, that

\footnotetext{
${ }^{4}$ The definitions are easily extended to pointed versions of ECGSs that are not required to be connected, but non-connected models are not particularly interesting in this context.
} 
the announcement $K_{i} \varphi$ can only be made when $K_{i} \varphi$ is in fact true. The choices open to a coalition is then the set of joint truthful public announcements its members can make.

Definition 4. The coalitional game corresponding to a pointed EGS $G S=\left(M, s, \varphi_{1}, \ldots, \varphi_{n}\right)$ is $\Gamma^{G S}=\left(N, \Omega, V, \sqsupseteq_{1}, \ldots, \sqsupseteq_{n}\right)$ where:

- $V(C)=\left\{M \mid \bigwedge_{i \in C} K_{i} \psi_{i}: \psi_{i} \in \mathcal{L}_{e l}, M, s \models K_{i} \psi_{i}\right\}$

- $\Omega=\bigcup_{C \in \mathcal{C}} V(C)$

- $M_{1} \sqsupseteq_{i} M_{2}$ iff $M_{2}, s \models \varphi_{i} \Rightarrow M_{1}, s \models \varphi_{i}$.

Outcomes are here models resulting from some (joint) announcement. We point out that while the choices of coalitions may "merely" seem to consist of all combinations of choices for the involved agents, this is not true because there is no trivial relationship between the consequences (i.e., potential satisfaction of goal formulae) of individual announcements and joint announcements.

We can thus view a pointed EGS as a coalitional game. We will sometimes abuse language and refer to the elements of $\Omega$ (the results of coalitional announcements) as announcements. Note that an agent can always make the trivial announcement $\left(K_{i} \top\right.$ is always true). Thus, $M \in V(C)$ for all $C$, and we call $M$ the non-informative announcement. An informative announcement is an $M^{\prime} \in V(C)$ such that $M^{\prime} \neq M$.

Example 2. Consider the initial information state $(M, t)$ in Example 1, and suppose that the goals for Ann and Bill are $\varphi_{1}=K_{1} q \vee K_{1} \neg q$ and $\varphi_{2}=K_{2} p \vee K_{2} \neg p-$ Ann wants to learn the truth about $q$ and Bill wants to learn the truth about $p$. This constitutes a pointed EGS $G S^{\prime}$. We now have that $\Omega=\left\{M, M\left|K_{1} p, M\right| K_{2} q, M \mid\left(K_{1} p \wedge K_{2} q\right)\right\}, V(\{1\})=$ $\left\{M, M \mid K_{1} p\right\}, V(\{2\})=\left\{M, M \mid K_{2} q\right\}$, and $V(\{1,2\})=\Omega$. Only $\left(M \mid\left(K_{1} p \wedge K_{2} q\right), t\right)$ realizes the goals of both players, $\left(M \mid K_{1} p, t\right)$ realizes the goal for Bill but not for Ann, and $\left(M \mid K_{2} q, t\right)$ realizes the goal for Ann but not for Bill.

The definition of the coalitional game corresponding to a pointed EGS is lifted to the epistemic coalitional game structure in the natural way.

Definition 5. The epistemic coalitional game structure corresponding to an EGS $G S=$ $\left(M, \varphi_{1}, \ldots, \varphi_{n}\right)$ where $M=\left(S, \sim_{1}, \ldots, \sim_{n}, \pi\right)$ is eg $=\left(S, N,\left\{\Gamma^{s}: s \in S\right\}, \sim_{1}\right.$ $\left., \ldots, \sim_{1}\right)$ where $\Gamma^{s}=\left(N, \Omega^{s}, V^{s}, \beth_{1}^{s}, \ldots, \beth_{n}^{s}\right)$ is the coalitional game corresponding to the pointed $\operatorname{EGS}\left(M, s, \varphi_{1}, \ldots, \varphi_{n}\right)$.

In the following we will implicitly assume, unless otherwise noted, that the model $M$ in the EGS we used to define a corresponding game structure, is connected.

\section{The Core}

Let us discuss the core of the corresponding games/game structures. We first investigate the core of coalitional games corresponding to pointed epistemic goal structures. An equivalent definition of the core for such games is as follows.

Lemma 2. Let $G S=\left(M, s, \varphi_{1}, \ldots, \varphi_{n}\right)$ be a pointed $E G S, \psi_{1}, \ldots, \psi_{n}$ be formulae and let $\Psi=\bigwedge_{i \in N} K_{i} \psi_{i} . M \mid \Psi$ is in the core of the corresponding coalitional game iff $M, s \mid=$ $\Psi$ and $\forall C \subseteq N \forall\left\{\beta_{j}: j \in C,(M, s) \models K_{j} \beta_{j}\right\} \exists i \in C\left(M\left|\bigwedge_{j \in C} K_{j} \beta_{j}, s \models \varphi_{i} \Rightarrow M\right| \Psi, s \models \varphi_{i}\right)$. 
A key property of a (general) coalitional game is whether the core is empty or not. In our case of a restricted class of games (the class of games corresponding to pointed EGSs are in fact a proper subset of all coalitional games, as will be shown formally in Section 8), it is however not prima facie clear that both cases actually occur. The next example shows that they do.

Example 3. Consider again $(M, t)$ from Example 1. Example 2 already presented a pointed EGS $G S^{\prime}=\left(M, t, K_{1} q \vee K_{1} \neg q, K_{2} p \vee K_{2} \neg p\right)$. In $\left(M \mid\left(K_{1} p \wedge K_{2} q\right), t\right)$ both goals are satisfied, so this model announcement is in the core. In $\left(M \mid K_{1} p, t\right)$, only agent 2 is satisfied, but agent 1 can do no better on her own, so also this announcement is in the core, and similarly for $\left(M \mid K_{2} q, t\right) . M$ is not in the core, because 1 and 2 can cooperate to make themselves both better off.

Let us now see how the core changes by varying the goals, while keeping the initial pointed epistemic structure constant.

1. Let $\varphi_{1}=\left(K_{2} p \vee K_{2} \neg p\right) \wedge \neg\left(K_{1} q \vee K_{1} \neg q\right)$ and $\varphi_{2}=\left(K_{1} q \vee K_{1} \neg q\right) \wedge \neg\left(K_{2} p \vee K_{2} \neg p\right)$. Then the core is empty. Note that neither goal is satisfied in $\left(M \mid\left(K_{1} p \wedge K_{2} q\right), t\right)$ or $(M, t)$, and that $\left(M \mid K_{1} p, t\right)$ satisfies $\varphi_{1}$ whereas $\left(M \mid K_{2} q, t\right)$ satisfies $\varphi_{2}$. The problem is that part of Ann's goal is to remain ignorant of the fact $q$ and that Bill has the ability to inform her about that, and vice versa. For example, suppose $M \mid K_{1} p$ was in the core. We have that $M \mid K_{1} p, t=\varphi_{1} \wedge \neg \varphi_{2}$, and that there is a subgroup of the set of all agents $\{1,2\}$, namely $\{2\}$, and an announcement for that agent, namely $K_{2} q$, such that $M \mid K_{2} q, t \models \varphi_{2}$ but $M \mid\left(K_{1} p \wedge \top\right), t \models \neg \varphi_{2}$. In other words, point 1 of Lemma 2 is violated.

2. Now swap the goals: $\varphi_{1}=\left(K_{1} q \vee K_{1} \neg q\right) \wedge \neg\left(K_{2} p \vee K_{2} \neg p\right)$ and $\varphi_{2}=\left(K_{2} p \vee\right.$ $\left.K_{2} \neg p\right) \wedge \neg\left(K_{1} q \vee K_{1} \neg q\right)$. The core is now $\left\{M\left|K_{1} p, M\right| K_{2} q\right\}$. Note that in this and the previous point, both goals cannot be satisfied simultaneously, as they contain complementary conjuncts.

3. Let $\varphi_{1}=K_{1} q \vee K_{1} \neg q$ and $\varphi_{2}=\top$. Clearly, $M$ is in the core. Because no epistemic operator in these goal formulas is in the scope of a negation, they are also satisfied in any further model restriction of $M$. Thus, the core is equal to $\Omega$.

The example above shows that the coalitional games we get from each pointed EGSs can have a range of different cores. However, this is far from the case for the epistemic (common knowledge) core in the epistemic coalitional game structure we get from the (non-pointed) EGS, as we now show.

Theorem 2. In an ECGS corresponding to an EGS, the epistemic core is either empty or the singleton set $\{M\}$ consisting of the non-informative announcement $M$.

Proof. Let $M=\left(S, \sim_{1}, \ldots, \sim_{n}, \pi\right)$ be an EGS and $\left(S, N,\left\{\Gamma^{s}: s \in S\right\}, \sim_{1}, \ldots, \sim_{1}\right)$ the corresponding ECGS. If $M^{\prime}$ is in the epistemic core then there is a coalition $C$ and a set of formulae $\left\{\psi_{i}: i \in C\right\}$ such that $M^{\prime}=M \mid \bigwedge_{i \in C} K_{i} \psi_{i}$ is in the core of $\Gamma^{s}$, for every $s \in S$. That means that $M^{\prime} \in V^{s}(C)$ for every $s$, and thus that $M, s \models K_{i} \psi_{i}$ for every $s$. But that means that $M^{\prime}=M \mid \bigwedge_{i \in C} K_{i} \psi_{i}=M$.

Corollary 1. In an ECGS corresponding to an EGS, the epistemic core is non-empty iff it is the singleton set containing the non-informative announcement. 
In other words, common knowledge of core-membership of some particular submodel implies not only that the initial model $M$ itself is in the core, but also that this is common knowledge, and furthermore that the initial model is the only submodel that can be commonly known to be in the core.

Theorem 3. In an ECGS corresponding to an $\operatorname{EGS}\left(M, \varphi_{1}, \ldots, \varphi_{n}\right)$, there is common knowledge of the core iff and only if:

1. for every $C$ and $s, V^{s}(C)=\{M\}$ (there are no informative announcements) ${ }^{5}$ and

2. for every $s \in S$, the core of $\Gamma^{s}$ is empty, or

3. (a) for every $s \in S$, the core of $\Gamma^{s}$ is the singleton set $\{M\}$ and

(b) $M \models \bigvee_{i \in N} \varphi_{i}$-the goal of at least one agent is already satisfied in the initial epistemic structure, and this is common knowledge

In other words, except in the uninteresting case that no agent can make any informative announcement in any state $\left(\Omega^{s}=\{M\}\right)$, there can only be common knowledge of the core if the core is empty or if it consists of only the initial model (corresponding to non-informative announcements by all agents). Furthermore, in the latter case it is common knowledge that at least one agent is satisfied in the initial model (but not necessarily who it is).

Example 4. Consider again the setting in Example 3. We saw that the core of the coalitional game corresponding to $\left(M, t, \varphi_{1}, \ldots, \varphi_{n}\right)$ was $\left\{M\left|K_{1} p, M\right| K_{2} q, M \mid\left(\left(K_{1} p \wedge\right.\right.\right.$ $\left.\left.K_{2} q\right)\right\}$. The reader can verify that the core corresponding to state $s$ and $u$ are, respectively, all subsets of $S$ containing $s$ and all subsets of $S$ containing $u$. Thus, in the epistemic coalitional game structure corresponding to $\left(M, \varphi_{1}, \ldots, \varphi_{n}\right)$, there is common knowledge of non-empty core but the epistemic core is empty, in other words it is commonly known that the core is non-empty but not that any particular announcement is in the core.

\subsection{Positive Goals}

A sufficient condition for a non-empty core in the game corresponding to a pointed EGS is that the goals are positive, i.e., in the following fragment of positive formulae: $\varphi::=p|\neg p| \varphi \wedge \varphi|\varphi \vee \varphi| K_{i} p$, where $p \in \Theta$. See Example 3 (initial goals as well as case 3). This notion of positive formulae is found, e.g., in [6]. The condition is not necessary for core-non-emptiness: a counterexample is Example 3.2. For a given structure $M$, the maximal informative announcement of a coalition $C$ is an $M^{\prime} \in V(C)$ which is a submodel of every $M^{\prime \prime} \in V(C)$. The maximal informative announcement always exists, and is unique.

Proposition 1 (Positive goals and the core). Let $G S=\left(M, s, \varphi_{1}, \ldots, \varphi_{n}\right)$ be a pointed EGS with positive goals. Then:

- The maximal informative announcement of the grand coalition is in the core.

\footnotetext{
${ }^{5}$ If the initial model $M$ is bisimulation contracted, this holds iff every $\sim_{i}$ is the universal relation.
} 
- If $M \mid \Psi$ is in the core and $M=\Psi^{\prime} \rightarrow \Psi$, then $M \mid \Psi^{\prime}$ is also in the core. In other words, if $M^{\prime}, M^{\prime \prime} \in V(N)$, then for all players $i: M^{\prime} \subseteq M^{\prime \prime}$ implies $M^{\prime} \sqsubseteq_{i} M^{\prime \prime}$. (And we even have that $M^{\prime} \subseteq M^{\prime \prime}$ iff $\left[M^{\prime} \sqsubseteq_{i} M^{\prime \prime}\right.$ for all players $\left.\left.i\right]\right)$.

- If the non-informative ann. is in the core, then all announcements are in the core.

Proposition 2 (Positive goals and the epistemic core). Let $G S=\left(M, \varphi_{1}, \ldots, \varphi_{n}\right)$ be an EGS with positive goals. Then:

1. There is common knowledge of non-empty core.

2. The epistemic core is non-empty iff the core in every state contains $M$ iff the core in every state contains all announcements

3. There is common knowledge of the core iff no informative ann. can be made.

\section{Logical Characterisations of Solution Concepts}

As mentioned in the introduction, an interesting problem is to find a logical formalism that can express key properties of games, such as solution concepts. Such a logic would allow agents to not only reason about what other agents know (epistemic logic), or what they can do (dynamic epistemic logic), but what rational agents will do. On one hand, there already exist logics for general coalitional games [3]. On the other hand, the use of such logics for purposes such as model checking, would require an explicit translation of epistemic goal structures to coalitional games. Of logics that are interpreted directly in epistemic structures, PAL seems not expressive enough since the solution concepts involve quantification over outcomes, but GAL seems like just the right thing since $\langle G\rangle$ corresponds exactly to existential quantification over $V(G)$. The logical characterisations of solution concepts in GAL given below are inspired by the corresponding characterisations in [3].

First observe that we can use the epistemic language to express preferences:

Lemma 3. Let $G S=\left(M, s, \varphi_{1}, \ldots, \varphi_{n}\right)$ be a pointed $E G S$. When $M \mid \Psi \in V(C)$ and $M \mid \Psi^{\prime} \in V\left(C^{\prime}\right)$ (in the corresponding game) for some $C, C^{\prime} \in \mathcal{C}$, we have that, for any $i \in N$ :

- $M\left|\Psi \sqsupseteq_{i} M\right| \Psi^{\prime}$ iff $M, s \mid=\left\langle\Psi^{\prime}\right\rangle \varphi_{i} \rightarrow\langle\Psi\rangle \varphi_{i}$

- $M\left|\Psi \exists_{i} M\right| \Psi^{\prime}$ iff $M, s \mid=\neg\left\langle\Psi^{\prime}\right\rangle \varphi_{i} \wedge\langle\Psi\rangle \varphi_{i}$

Let us move on to the core. Let, when $\Psi, \varphi_{1}, \ldots, \varphi_{n}$ are formulae,

$$
\operatorname{INCORE}\left(\Psi, \varphi_{1}, \ldots, \varphi_{n}\right)=\Psi \wedge \bigwedge_{C \subseteq N}\left(\langle C\rangle \bigwedge_{i \in C} \varphi_{i} \rightarrow\langle\Psi\rangle \bigvee_{i \in C} \varphi_{i}\right)
$$

Proposition 3. Let $G S=\left(M, s, \varphi_{1}, \ldots, \varphi_{n}\right)$ be a pointed $E G S$, and $\psi_{1}, \ldots, \psi_{n} \in \mathcal{L}_{e l}$. Let $\Psi=\bigwedge_{i \in N} K_{i} \psi_{i}$. Then $M \mid \Psi$ is in the core of the corresponding coalitional game iff $M, s=\operatorname{INCORE}\left(\Psi, \varphi_{1}, \ldots, \varphi_{n}\right)$.

Proposition 3 lets us check whether a particular announcement is in the core. However, this might not be known by the agents involved. The following proposition lets us check whether or not the agents have common knowledge of the fact that some announcement is in the core (it follows immediately from Corollary 1). Note that $C_{N} \varphi$ means that $N$ have common knowledge of $\varphi$. 
Proposition 4. Let $G S=\left(M, \varphi_{1}, \ldots, \varphi_{n}\right)$ be a connected $E G S$, and $\psi_{1}, \ldots, \psi_{n} \in \mathcal{L}_{e l}$. Then the epistemic core of the corresponding epistemic coalitional game structure is non-empty iff for an arbitrary state $s,(M, s)=C_{N} \operatorname{INCORE}\left(\top, \varphi_{1}, \ldots, \varphi_{n}\right)$.

While we are not able to express the property that the core is (non-)empty in a similar way to $\operatorname{INCORE}(\cdot)$ in GAL (and conjecture that it is indeed not possible), this property can be expressed in a weaker sense. For any finite pointed EGS (a structure with a finite number of states), $V(i)$ will be finite for each agent $i$. Let, then, for each $u \in V(i), \varphi_{u}$ be a formula such that $u=M \mid K_{i} \varphi_{u}$, and:

$$
C E=\bigwedge_{u_{1} \in V(1), \cdots, u_{n} \in V(n)}\left(\bigwedge_{i \in N} K_{i} \varphi_{u_{i}} \rightarrow \neg \operatorname{INCORE}\left(\bigwedge_{i \in N} \varphi_{u_{i}}, \varphi_{1}, \ldots, \varphi_{n}\right)\right)
$$

We then have that the core of the pointed EGS is empty iff $M, s \mid=C E$. Note that the expression $C E$ (unlike INCORE) depends on the particular model, but the formulae $\varphi_{u}$ can easily be derived from the model. This is easily extended to express common knowledge of empty core (assuming connectedness):

$$
C K C E=C_{N} \bigwedge_{u_{1} \in U(1), \cdots, u_{n} \in U(n)}\left(\bigwedge_{i \in N} K_{i} \varphi_{u_{i}} \rightarrow \neg \operatorname{INCORE}\left(\bigwedge_{i \in N} \varphi_{u_{i}}, \varphi_{1}, \ldots, \varphi_{n}\right)\right)
$$

where for each $i, U(i)=\bigcup_{t \in S} V^{t}(i)$ and $V^{t}$ is the characteristic function of the coalitional game corresponding to state $t$. Now, $M, s=C K C E$ (for any $s$ ) iff there is common knowledge of non-empty core.

\section{Representation and Compactness}

Which coalitional games correspond to pointed epistemic goal structures? Not all. For example, a coalitional game where $\omega \in V(1)$ but $\omega \notin V(\{1,2\})$ cannot correspond to any pointed EGS, because if it did it would necessarily be coalition monotonic: any announcement by a coalition can also be made by a superset of that coalition (the "new" agents can merely make the trivial announcement).

In this section, we study this question for finite games. We define a class of coalitional announcement games (Def. 6), and show (Prop. 5 and Th. 4) that, up to bisimulation equivalence, a finite coalitional game is a coalitional announcement game if and only if it corresponds to a pointed epistemic goal structure.

Definition 6. A (finite) coalitional announcement game is a finite coalitional game $\Gamma=$ $\left(N, \Omega, V, \sqsupseteq_{1}, \ldots, \sqsupseteq_{n}\right)$ where whenever $i, j \in N, C, C^{\prime} \subseteq N$ and $\omega \in \Omega$ :

1. If $C \subseteq C^{\prime}$ then $V(C) \subseteq V\left(C^{\prime}\right)$ (coalition monotonicity)

2. There is an integer $m$ such that $|V(i)|=2^{m}$ (combinations of individual ann.)

3. For $|C|>1,\left|V(C) \backslash \bigcup_{C^{\prime} \subset C, C^{\prime} \neq \emptyset} V\left(C^{\prime}\right)\right| \leq \times_{i \in C}(|V(i)|-1)$

4. $\bigcap_{C \in \mathcal{C}} V(C) \neq \emptyset$ (trivial ability)

5. If $|V(i)| \geq 4$ and $V(i) \backslash\{\omega\} \subseteq V(j)$, then $V(i) \subseteq V(j)$ 
6. Either (a) $\omega \sqsupseteq_{i} \omega^{\prime}$ for all $\omega, \omega^{\prime} \in \Omega$ ( $i$ is indifferent between all outcomes), or $(b)$ $\Omega=\operatorname{Good}_{i} \uplus \operatorname{Bad}_{i}$, such that ${ }^{6}$ for all $\omega_{g} \in \operatorname{Good}_{i}$ and $\omega_{b} \in \operatorname{Bad}_{i} \omega_{g} \sqsupset_{i} \omega_{b}$, and for $X \in\{$ Good,Bad $\}$ and all $\omega, \omega^{\prime} \in X, \omega \sqsupseteq_{i} \omega^{\prime}$ and $\omega^{\prime} \sqsupseteq_{i} \omega$

Proposition 5. A finite coalitional game that corresponds to a pointed epistemic goal structure is a coalitional announcement game.

The next theorem shows that all coalitional announcement games correspond, up to bisimulation equivalence, to pointed EGSs. An interesting additional result is compactness: every such game (i.e., every coalitional game having the properties listed in Def. 6) can be represented by a Kripke structure of exponentially smaller size.

Theorem 4. Any finite coalitional announcement game $\Gamma=\left(N, \Omega, V, コ_{1}, \ldots, \sqsupseteq_{n}\right)$ is bisimulation equivalent to the coalitional game corresponding to some pointed epistemic goal structure with $\sum_{i \in N} \log _{2}|V(i)|+1$ states.

Proof. We leave out some details due to lack of space. Given a coalitional announcement game $\Gamma=\left(N, \Omega, V, \sqsupseteq_{1}, \ldots, \sqsupseteq_{n}\right)$, we construct a pointed EGS $G S=\left(M, s, \varphi_{1}, \ldots, \varphi_{n}\right)$ where $M=\left(S, \sim_{1}, \ldots, \sim_{n}, \pi\right)$. For each $i$, let $m_{i} \geq 0$ be such that $|V(i)|=2^{m_{i}}$ (Def. 6.2). We first construct $M$ :

- For each $i$, let $S_{i}=\left\{s_{1}^{i}, \ldots, s_{m_{i}}^{i}\right\}$ be a set of $m_{i}$ states and let $P_{i}=\left\{p_{1}^{i}, \ldots, p_{m_{i}}^{i}\right\} \subseteq$ $\Theta$ be a collection of $m_{i}$ atoms, such that $S_{i} \cap S_{j}=\emptyset$ and $P_{i} \cap P_{j}=\emptyset$ when $i \neq j$

$-S=\left\{s_{0}\right\} \cup \bigcup_{i \in N} S_{i}$

$-s=s_{0}$

- $\pi\left(p_{0}\right)=\left\{s_{0}\right\}, \pi\left(p_{k}^{i}\right)=\left\{s_{k}^{i}\right\}$, and $\pi(p)=\emptyset$ for other $p \in \Theta$

- For each agent $i$, let $\sim_{i}$ be an equivalence relation on $S$ s.t.

- $s_{0} \sim_{i} s$ iff $s \notin S_{i} \quad$ and $s_{j}^{i} \sim_{i} s^{\prime}$ iff $s^{\prime}=s_{j}^{i}$, for any $s_{j}^{i} \in S_{i}$ and $s^{\prime} \in S$

For each agent $i, \sim_{i}$ partitions $S$ into $m_{i}+1$ equivalence classes $\left[s_{0}\right]_{\sim_{i}},\left\{s_{1}^{i}\right\}, \ldots,\left\{s_{m_{i}}^{i}\right\}$ (where only the first one is not necessarily a singleton). In state $s_{0}$, agent $i$ can eliminate the equivalence class $\left\{s_{1}^{i}\right\}$ by announcing $K_{i} \neg p_{1}^{i}-$ this formula is false in, and only in, state $s_{1}^{i}$. Similarly for the other equivalence classes (except $\left[s_{0}\right]_{\sim_{i}}$ ), and combinations of them. Let $A(i)=\left\{\neg x: x \in P_{i}\right\}$. The set $\left\{K_{i} a: a \in A(i)\right\}$ represents the "atomic" announcements $i$ can make. Each announcement $i$ can make in $s$ will eliminate zero, one, or several of the equivalence classes $\left\{s_{1}^{i}\right\}, \ldots,\left\{s_{m_{i}}^{i}\right\}$, and corresponds to a set $\alpha \subseteq A(i)$ ( $i$ announces $K_{i} \bigwedge \alpha$, which eliminates $\left\{s_{k}^{i}\right\}$ iff $\neg p_{k}^{i} \in \alpha$ ). When $\alpha$ is a set of formulae, we will use the notation $\bar{\alpha}$ for $\bigwedge \alpha$. For a coalition $C \in \mathcal{C}$, a set $A_{C}=\left\{\alpha_{i} \subseteq\right.$ $A(i): i \in C\}$ corresponds to one of the announcement $M \mid \bigwedge_{i \in C} K_{i} \overline{\alpha_{i}}$ that a coalition can make come about. Let $\Delta_{C}$ denote the set of all such $A_{C}$. We will use $M \mid A_{C}$ for $M \mid \bigwedge_{i \in C} K_{i} \overline{\alpha_{i}}$ to simplify notation. Furthermore, let $\operatorname{supp}\left(A_{C}\right)=\left\{i \in C: \alpha_{i} \neq 0\right\}$.

Let $\Gamma^{G S}=\left(N, \Omega^{\prime}, V^{\prime}, \sqsupseteq_{1}^{\prime}, \ldots, \sqsupseteq_{n}^{\prime}\right)$ be the game corresponding to $G S$. We haven't specified the goals $\varphi_{i}$ yet; we will first establish some properties of $\Gamma^{\prime}$ which do not depend on the goals. It is easy to see that:

$$
V^{\prime}(C)=\left\{M \mid A_{C}: A_{C} \in \Delta_{C}\right\}
$$

\footnotetext{
${ }^{6} \uplus$ denotes disjoint union.
} 
It can easily be shown that all the members of $\Delta_{C}$ represent different submodels:

$$
\forall A_{C}, B_{C} \in \Delta_{C},\left(M\left|A_{C}=M\right| B_{C}\right) \Rightarrow\left(A_{C}=B_{C}\right)
$$

It can also easily be shown that different coalitions can do the same model update, but only if they only differ by agents merely making the trivial announcement: $M \mid A_{C_{1}}=$ $M \mid A_{C_{2}} \Rightarrow \operatorname{supp}\left(A_{C_{1}}\right)=\operatorname{supp}\left(A_{C_{2}}\right)$.

We will construct a full bisimulation between $\Gamma$ and $\Gamma^{G S}$. For any $C \in \mathcal{C}$, let $X_{C}=V^{\prime}(C) \backslash \bigcup_{C^{\prime} \subset C, C^{\prime} \neq \emptyset} V^{\prime}\left(C^{\prime}\right)$ and $Y_{C}=V(C) \backslash \bigcup_{C^{\prime} \subset C, C^{\prime} \neq \emptyset} V\left(C^{\prime}\right)$. It is easy to see that, when $C$ is not a singleton, $\left|X_{C}\right|=\times_{i \in C}\left(2^{m_{i}}-1\right)$. This is the maximum number of "new" announcements $C$ can make that a proper subcoalition cannot make, and it is equal to the number of possible joint announcements where every member of $C$ makes an informative announcement. Each agent can make $\left|V^{\prime}(i)\right|-1=2^{m_{i}}-1$ such announcements. By Def. 6.3, $|X| \geq|Y|$. When $C=\{i\}$ is a singleton, $X_{i}=V^{\prime}(i)$ and $Y_{i}=V(i)$, and $\left|X_{C}\right|=\left|V^{\prime}(i)\right|=2^{m_{i}}=|V(i)|=\left|Y_{C}\right|$. Let $\omega_{0}$ be such that $\omega_{0} \in \bigcap_{C \in \mathcal{C}} V(C)$ (exists by Def. 6.3). Let $g_{C}: X_{C} \rightarrow Y_{C}$ be a function such that (i) if $M \in X_{C}$ then $g_{C}(M)=\omega_{0}$ and (ii) $g_{c}$ is surjective. Such a function exists because $\left|X_{C}\right| \geq\left|Y_{C}\right|$.

For each $C \in \mathcal{C}$ we define a function $f_{C}: V^{\prime}(C) \rightarrow V(C)$ as follows. As an auxiliary definition, let $f_{\emptyset}(\omega)=\omega_{0}$ for any $\omega \in \Omega^{\prime}$.

$$
f_{C}\left(M \mid A_{C}\right)= \begin{cases}f_{\operatorname{supp}\left(A_{C}\right)}\left(M \mid A_{C}\right) & \operatorname{supp}\left(A_{C}\right) \neq C \\ g_{C}(\omega) & \text { otherwise }\end{cases}
$$

We argue that $f_{C}$ is well defined. Given an $\omega^{\prime} \in V^{\prime}(C)$, by (2) there is a unique $A_{C}$ such that $\omega^{\prime}=M \mid A_{C}$, so $\operatorname{supp}\left(A_{C}\right)$ is well defined. In the case that $\operatorname{supp}\left(A_{C}\right) \neq C$, the definition says that $f\left(M \mid A_{C}\right)=f_{\text {supp }\left(A_{C}\right)}\left(M \mid A_{C}\right)$, which requires that (i) $M \mid A_{C} \in$ $V^{\prime}\left(\operatorname{supp}\left(A_{C}\right)\right)$ and (ii) $f_{\operatorname{supp}\left(A_{C}\right)}\left(M \mid A_{C}\right) \in V(C)$. (i) follows from the fact that $M \mid A_{C}=$ $M \mid \operatorname{supp}\left(A_{C}\right)$. (ii) follows from coalition monotonicity for $\Gamma$ (Def. 6.1) since $\operatorname{supp}\left(A_{C}\right) \subset$ $C$. Note that when $\operatorname{supp}\left(A_{C}\right)=\emptyset, \operatorname{supp}\left(A_{C}\right) \neq C$ and $f_{C}\left(M \mid A_{C}\right)=\omega_{0}$.

It can now be shown that:

$$
\omega \in V^{\prime}\left(C_{1}\right) \cap V^{\prime}\left(C_{2}\right) \Rightarrow f_{C_{1}}(\omega)=f_{C_{2}}(\omega)
$$

and that $f_{C}$ is surjective for all $C \in \mathcal{C}$.

We now construct a surjective function $f: \Omega^{\prime} \rightarrow \Omega$ as follows: $f\left(M \mid A_{C}\right)=$ $f_{C}\left(M \mid A_{C}\right)$. (3) guarantees that $f$ is well defined. Surjectivity of $f$ follows immediately from surjectivity of each $f_{C}$ : if $\omega \in \Omega=\bigcup_{C \in \mathcal{C}} V(C) ; \omega \in V(C)$ for some $C \in \mathcal{C}$; $f_{C}\left(\omega^{\prime}\right)=\omega$ for some $\omega^{\prime} \in V^{\prime}(C) ; f\left(\omega^{\prime}\right)=f_{C}\left(\omega^{\prime}\right)=\omega$.

Finally, we define goals. To do that, we need to syntactically describe the exact consequences of announcements. When $\alpha \subseteq A(i)$, and $C \in \mathcal{C}$ and $A_{C}=\left\{\alpha_{i} \subseteq A(i): i \in\right.$ $C\} \in \Delta_{C}$ let: $\chi_{i}(\alpha)=\bigwedge_{j \in N} K_{j} \bar{\alpha} \wedge \bigwedge_{j \in N, j \neq i} \bigwedge_{x \in A(i) \backslash \alpha} \neg K_{j} x$ and $\chi_{C}\left(A_{C}\right)=\bigwedge_{i \in C} \chi_{i}\left(\alpha_{i}\right)$. The first main conjunct in $\chi_{i}(\alpha)$ says that the $i$-equivalence classes described by $\alpha$ have been removed; the second that no other $i$-equivalence classes have been removed. It is easy to see that for any $A_{C}, B_{C} \in \Delta_{C}, M \mid A_{C}, s \models \chi_{C}\left(B_{C}\right)$ iff $A_{C}=B_{C}$.

Let $i$ be an agent. From Def. 6.6 we have that $i$ is either indifferent between all outcomes or partitions $\Omega$ into $\operatorname{Good}_{i}$ and $\mathrm{Bad}_{i}$. In the former case, let $\varphi_{i}=\perp$, in the 
latter let: $\varphi_{i}=\bigvee_{C \in \mathcal{C}, A_{C} \in \Delta_{C}, f\left(M \mid A_{C}\right) \in \text { Good }_{i}} \chi_{C}\left(A_{C}\right)$. Let $Z \subseteq \Omega \times \Omega^{\prime}$ be defined as follows: $\omega Z \omega^{\prime} \Leftrightarrow f\left(\omega^{\prime}\right)=\omega$. It can be shown that $Z$ is a full bisimulation between $\Gamma^{G S}$ and $\Gamma$.

Together, Prop. 5 and Th. 4 answer the question: which coalitional games correspond to epistemic goal structures and which do not? Thus we know the properties of the individual games that can be played, and of the games that cannot be played.

An even more interesting question is: which epistemic coalitional game structures correspond to (non-pointed) epistemic goal structures? Although each individual game in each state will be a coalitional announcement game, the results presented here do not answer that question: not all combinations of coalitional announcement games, i.e., epistemic coalitional game structures consisting of only coalitional announcement games, correspond to epistemic goal structures; such structures also have interaction properties between games in different states. This is an open question for future work.

\section{Discussion}

Motivated by analysing rational behaviour, and in particular coalitional stability, in information-(ex)changing settings, in this paper we identified coalitional games inherent in standard epistemic structures extended with a simple notion of epistemic goals. We introduced a new, but very natural from a logical and computational viewpoint, qualitative model of coalitional games under imperfect information, and identified different variants of common knowledge related to the core of such games. We characterised in several different ways the games corresponding to epistemic structures and their solution properties. The representation theorem completely characterises the properties of (finite) games being played in such settings. Along the way, we introduced a bisimulation notion for coalitional games. The other side of the coin of the representation theorem is that epistemic goal structures are a compact representation of a certain class of coalitional games, i.e., finite coalitional announcement games. Compact representations of coalitional games is of great interest in multi-agent systems [9].

The most prominent related work is cooperative Boolean games (CBGs) [9]. In standard Boolean games [18, 12], each agent has an individual goal in the form of a propositional formula, and available actions correspond to the agent controlling some of the Boolean variables. CBGs model a similar setting as coalitional games. In coalitional announcement games an agent, rather than controlling Boolean variables, "controls" common knowledge of any formula he or she knows. In CBGs there is an additional assumption that each action has a cost, and solution concepts are derived under the assumption that an agent tries to achieve her goals while minimising the cost. Key results are characterisations of computational complexity. While the cost-based concepts are not directly applicable to the current setting, it is quite possible that some of the complexity results in [9] might shed light on complexity problems in the current setting, a research issue we haven't addressed in the current paper; an interesting opportunity for future work. The probably most interesting direction for future work is to extend the representation result in Section 8 to the case of epistemic coalitional game structures. Relaxing the assumptions discussed in the introduction also give plentiful opportunities for future work. We see two main, orthogonal directions: more general dynamic epistemic logics and more sophisticated preference/goal models. 


\section{References}

1. T. Ågotnes, P. Balbiani, H. van Ditmarsch, and P. Seban. Group announcement logic. Journal of Applied Logic, 8(1):62-81, 2010.

2. T. Ågotnes, J. van Benthem, H. van Ditmarsch, and S. Minica. Question-answer games. Journal of Applied Non-Classical Logics, 21(3-4):265-288, 2011.

3. T. Ågotnes, W. van der Hoek, and M. Wooldridge. Reasoning about coalitional games. Artificial Intelligence, 173(1):45-79, 2009.

4. T. Ågotnes and H. van Ditmarsch. What will they say? - public announcement games. Synthese (Special Section on Knowledge, Rationality and Action), 179(1):57-85, 2011.

5. P. Balbiani, A. Baltag, H. van Ditmarsch, A. Herzig, T. Hoshi, and T. D. Lima. What can we achieve by arbitrary announcements? A dynamic take on Fitch's knowability. In D. Samet, editor, Proceedings of TARK XI, pages 42-51, 2007.

6. P. Balbiani, A. Baltag, H. van Ditmarsch, A. Herzig, T. Hoshi, and T. D. Lima. 'Knowable' as 'known after an announcement'. Review of Symbolic Logic, 1(3):305-334, 2008.

7. G. Chalkiadakis and C. Boutilier. Bayesian reinforcement learning for coalition formation under uncertainty. In Proceedings of AAMAS 2004, pages 1090-1097. 2004.

8. G. Chalkiadakis, E. Elkind, and N. R. Jennings. Simple coalitional games with beliefs. In Proceedings of JCAI 2009, pages 85-90. 2009.

9. P. Dunne, W. van der Hoek, S. Kraus, and M. Wooldridge. Cooperative boolean games. In Proceedings of AAMAS 2008, pages 1015-1022. 2008.

10. R. Fagin, J. Halpern, Y. Moses, and M. Vardi. Reasoning about Knowledge. MIT Pr. 1995.

11. J. Gerbrandy and W. Groeneveld. Reasoning about information change. J. of Logic, Language, and Inform., 6:147-169, 1997.

12. P. Harrenstein. Logic in Conflict. PhD thesis, Utrecht University, 2004.

13. J. C. Harsanyi. Games with Incomplete Information Played by 'Bayesian' Players, Parts I, II, and III. Management Science, 14:159-182, 320-334, 486-502, 1967-1968.

14. S. Ieong and Y. Shoham. Bayesian coalitional games. In Proc. of AAAI, pages 95-100. 2008.

15. J.-J. Meyer and W. van der Hoek. Epistemic Logic for AI and Computer Science. Cambridge University Press, 1995.

16. R. B. Myerson. Virtual utility and the core for games with incomplete information. Journal of Economic Theory, 136(1):260-285, 2007.

17. M. Osborne and A. Rubinstein. A Course in Game Theory. MIT Press, 1994.

18. J.-J. M. P. Harrenstein, W. van der Hoek and C. Witteveen. Boolean games. In Proceeding of TARK VIII, pages 287-298, 2001.

19. J. Plaza. Logics of public communications. In Proceedings of the 4th International Symposium on Methodologies for Intelligent Systems, pages 201-216, 1989.

20. J. van Benthem. What one may come to know. Analysis, 64(2):95-105, 2004.

21. W. van der Hoek and M. Pauly. Modal logic for games and information. In The Handbook of Modal Logic, pages 1180-1152. Elsevier, 2006.

22. H. van Ditmarsch, W. van der Hoek, and B. Kooi. Dynamic Epistemic Logic. Springer, 2007. 